\title{
The use of parabolic mirrors in combined low-coherence and confocal refractive index measurement
}

\author{
Daniel Francis, Helen D. Ford, Jonathan M. Hallam, and Ralph P. Tatam* \\ Engineering Photonics, Cranfield University, Bedford, MK43 0AL, UK
}

\begin{abstract}
Low-coherence interferometry is combined with confocal scanning to provide remote refractive index and thickness measurements of transparent materials. The influence of lens aberrations in the confocal measurement is assessed through investigation of the axial point-spread functions (APSFs) generated using optical configurations comprised of paired aspherics and paired achromats. Off-axis parabolic mirrors are suggested as an alternative to lenses and are shown to exhibit much more symmetric APSFs provided the system numerical aperture is not too high. Refractive index and thickness measurements are made with each configuration with most mirror pairings offering better than twice the repeatability and accuracy of either lens pairing.
\end{abstract}

Keywords: confocal, low-coherence, interferometry, refractive index, parabolic mirror, aberration, point-spread function

\section{INTRODUCTION}

Low-coherence interferometry [1] is a technique that offers great potential for remote refractive index measurement giving it an advantage over alternative methods that require sample contact such as Abbe refractometry [2] or fibre-optic probes [3]. Low-coherence interferometry can measure the group refractive index and optical thickness of translucent media however, if the phase index and physical thickness are required, additional measurements are needed. Confocal scanning is a convenient complementary technique which involves scanning the focused illumination beam through each interface of the sample under test [4]. To acquire all three variables; the phase and group refractive indices and the physical thickness, the low-coherence and confocal measurements need to be combined via a measure of the sample's chromatic dispersion [5]. This can be done by making the confocal measurements at multiple wavelengths.

The dispersion measurement can be made using multiple sources to make the confocal measurement [6] or in a single optical instrument comprising a broadband source and a spectrometer [7]. The variation in the confocal measurement with wavelength is often very small, typically around $1 \mu \mathrm{m}$ for a $50 \mathrm{~nm}$ bandwidth source for most optical glasses. A very accurate determination of the confocal peak locations is therefore required. The confocal peak shapes can be distorted due to imaging aberrations and this can cause errors in the determination of the peak's centre. The numerical aperture (NA) of the overall system also has an effect, with high NA systems often being subject to greater levels of aberration and low NA systems resulting in broad peak profiles which limit the thickness of objects that can be measured due to peak overlap.

The confocal peaks observed correspond to the axial point-spread function (APSF) of the optical system being used. In this paper, we analyse the APSFs generated using different commercial off-the-shelf lens combinations for both surfaces of a BK7 window. Asymmetry of the confocal peaks generated with each of the lens pairings is due to spherical aberration and this introduces uncertainty into the refractive index measurement due to increased difficulty in accurately locating the peak centre. Off-axis parabolic (OAP) mirrors are generally free of spherical aberration and can offer improved beam collimation and focusing. Replacing the lens pairings in the optical system with OAP mirrors results in much more symmetric APSFs provided the system numerical aperture (NA) is not too high. Refractive index and thickness measurements are made with two different lens pairings and four different OAP mirror pairings. Three of the OAP mirror pairings presented here yield significant improvement in accuracy and repeatability over either of the lens pairings, with the exception being due to high NA of the pairing.

*Author contact: r.p.tatam@cranfield.ac.uk 


\section{REFRACTIVE INDEX MEASUREMENT PRINCIPLES}

This section briefly describes the refractive index and thickness measurement procedure. A more detailed explanation is available in [6] or [7]. The confocal measurement is made by scanning the beam (or object) through focus and detecting the reflected light. As the scan is made, a peak is observed in the integrated signal for each of the object's interfaces. The separation of the peaks $\Delta z$ is a quantity which is dependent on the phase refractive index $n_{\mathrm{p}}$, the physical separation of the interfaces $t$ and the NA of the optical system, as given by

$$
\Delta z=t \times \frac{\sqrt{n_{\mathrm{air}}^{2}-N A^{2}}}{\sqrt{n_{\mathrm{p}}^{2}-N A^{2}}}
$$

The low-coherence measurement is made by locating the focus at each interface in turn and scanning a reference mirror located in the other arm of the interferometer. A burst of interference fringes is observed in the detected signal when the separation of optical paths in the interferometer is within the coherence length of the source. The separation of the fringe envelopes $\Delta l$ is dependent on the interface separation and the group refractive index $n_{\mathrm{g}}$, given by

$$
\Delta l=t \times n_{\mathrm{g}}
$$

Equations (1) and (2) can be combined using the expression for chromatic dispersion

$$
n_{\mathrm{g}}(\nu)=n_{\mathrm{p}}(\nu)+\nu \frac{\mathrm{d} n_{\mathrm{p}}(\nu)}{\mathrm{d} \nu}
$$

where $\nu$ is the optical frequency. For low NA, this results in [6]

$$
t^{2}=\frac{\Delta l \Delta z}{1-\frac{\nu}{\Delta z} \frac{\mathrm{d} \Delta z}{\mathrm{~d} \nu}}
$$

Usually, the NA is high enough that this approximation cannot be used without introducing appreciable error. The NA dependent equation can be solved using a polynomial expansion given in [6] and [7]. The expression is dependent on the term $\mathrm{d} \Delta z / \mathrm{d} \nu$, which is the dispersion of the confocal parameter with respect to frequency and can be measured by making the confocal measurement at multiple wavelengths. Once $t$ has been calculated, $n_{\mathrm{p}}$ and $n_{\mathrm{g}}$ can be readily obtained from equations (1) and (2).

\section{EXPERIMENTAL ASSESSMENT OF CONFOCAL CONFIGURATIONS}

\subsection{Setup of measurement system}

The experimental layout of the measurement system is shown in figure 1. The interferometer is composed of a broadband fibre-optic coupler (Thorlabs TW850R2A2) and a super-luminescent diode (SLD) centred at $840 \mathrm{~nm}$ with $50 \mathrm{~nm}$ bandwidth (Superlum M-T-850-HP-I). The outputs of the coupler are terminated with $8^{\circ}$ angle polished connectors. Light on the return leg of the coupler is detected using a line-scan spectrometer (Bayspec OCT spectrograph @850) which consists of a diffraction grating and a line-scan camera (Basler spL4096-140km) with a 4,096 pixel array, $140 \mathrm{kHz}$ maximum acquisition rate, and 12-bit digital resolution. The reference mirror and a focusing lens, and the test object are mounted on a pair of precision linear translation stages (Physik Instrumente V408) which have a maximum travel of $50 \mathrm{~mm}$, a maximum velocity of $500 \mathrm{~mm} / \mathrm{s}$, and a minimum incremental motion of $20 \mathrm{~nm}$.

Measurements were made using either paired lenses or paired OAP mirrors in the confocal channel and the alignment of these two types of configuration are shown in the two insets in figure 1. Alignment of OAP mirrors can be challenging and was aided using a shear-plate interferometer (SPI). Successful collimation of the beam is indicated when straight line fringes are observed through the SPI aligned parallel to a groove indented into the screen of the SPI. The test object used in each of the measurements was a BK7 window that was nominally $3 \mathrm{~mm}$ thick. 


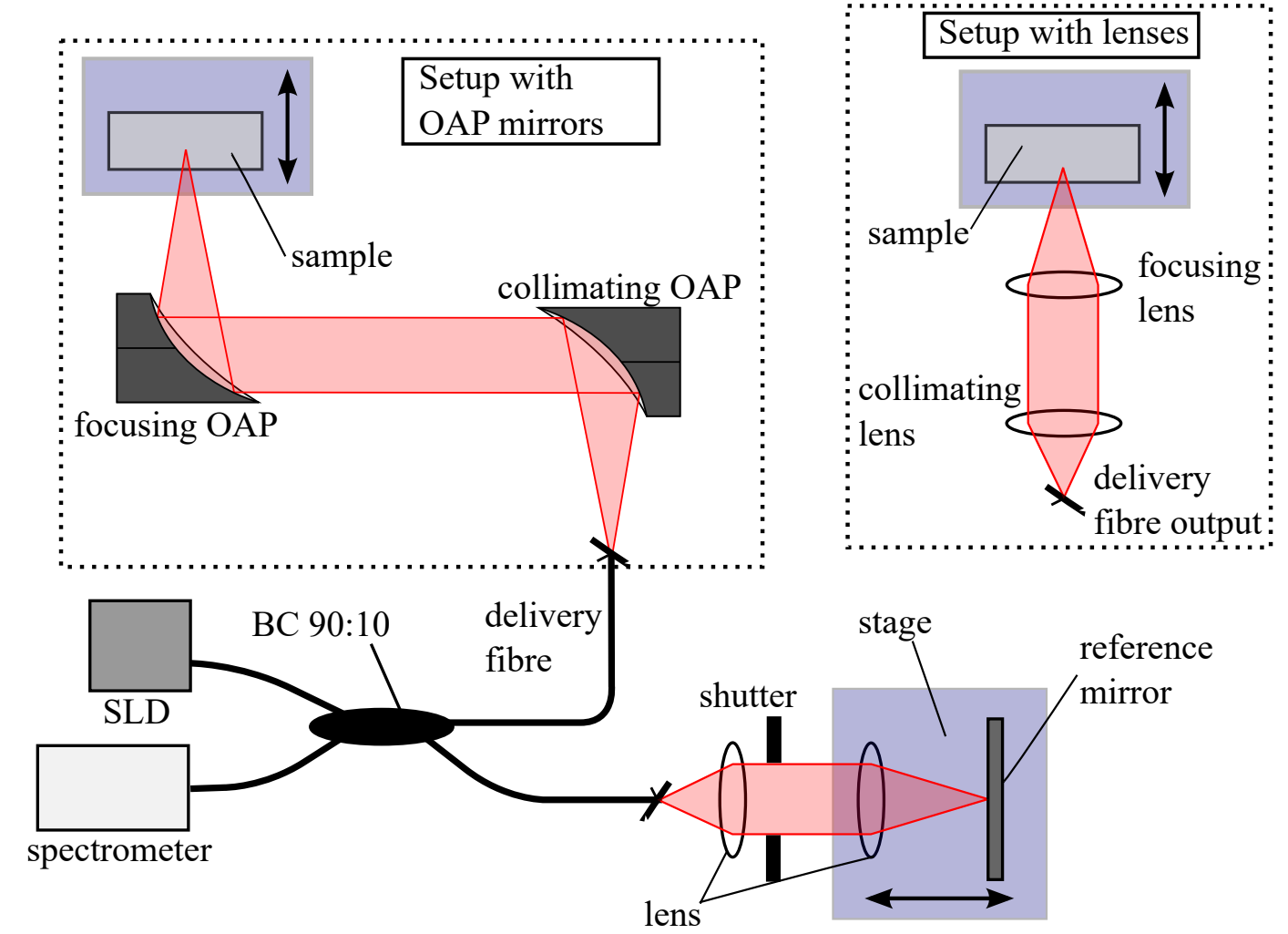

Figure 1. Schematic showing the configuration of the experimental system. SLD is the super-luminescent diode, BC is the broadband coupler, OAP = off-axis parabolic mirror. The blue shading indicates which components are mounted on the translation stages. The insets show the configuration used when confocal measurements are made using either paired OAP mirrors or lenses.

\subsection{Analysis of axial PSFs}

Two different lens configurations and four different OAP mirror configurations with different system NAs were tested. The lens pairings were either achromats or apsherics. The achromats were Thorlabs AC080-020-B-ML with $20 \mathrm{~mm}$ focal length used to collimate and AC080-016-B-ML with $16 \mathrm{~mm}$ focal length used to focus and the aspherics were a pair of Newport 5725-B-H lenses. The lenses were anti-reflection coated with an effective range of $650-1050 \mathrm{~nm}$. The numerical apertures of the two configurations were 0.150 for the achromats and 0.121 for the aspherics. The confocal peaks generated for each of the lens pairings are shown in figure 2. The front and rear peaks obtained with the achromats are shown in figure 2 (a) and (b) respectively and the front and rear peaks obtained with the aspherics are shown in figure 2 (c) and (d) respectively. The experimental data is shown as a thick red curve and the least-squares fit to the axial point-spread function (APSF) is shown as thinner black curves. The functional form of the diffraction limited APSF, $h$, for non-scattering media is given by [8]

$$
h(d)=\frac{1}{\left(\frac{d}{z}\right)^{2}+1}
$$

where $d$ is the focal shift and

$$
z=\frac{\pi n_{\mathrm{p}} \omega_{0}}{\lambda}
$$

where $\omega_{0}$ is the beam waist and $\lambda$ is the wavelength. There is noticeable asymmetry in each of the experimentally obtained peaks in figure 2 and significant divergence from the APSF fit for each of the peaks. Also, a small side lobe located to the left of the main peak appears for both front and rear surfaces when using the achromatic lenses. 

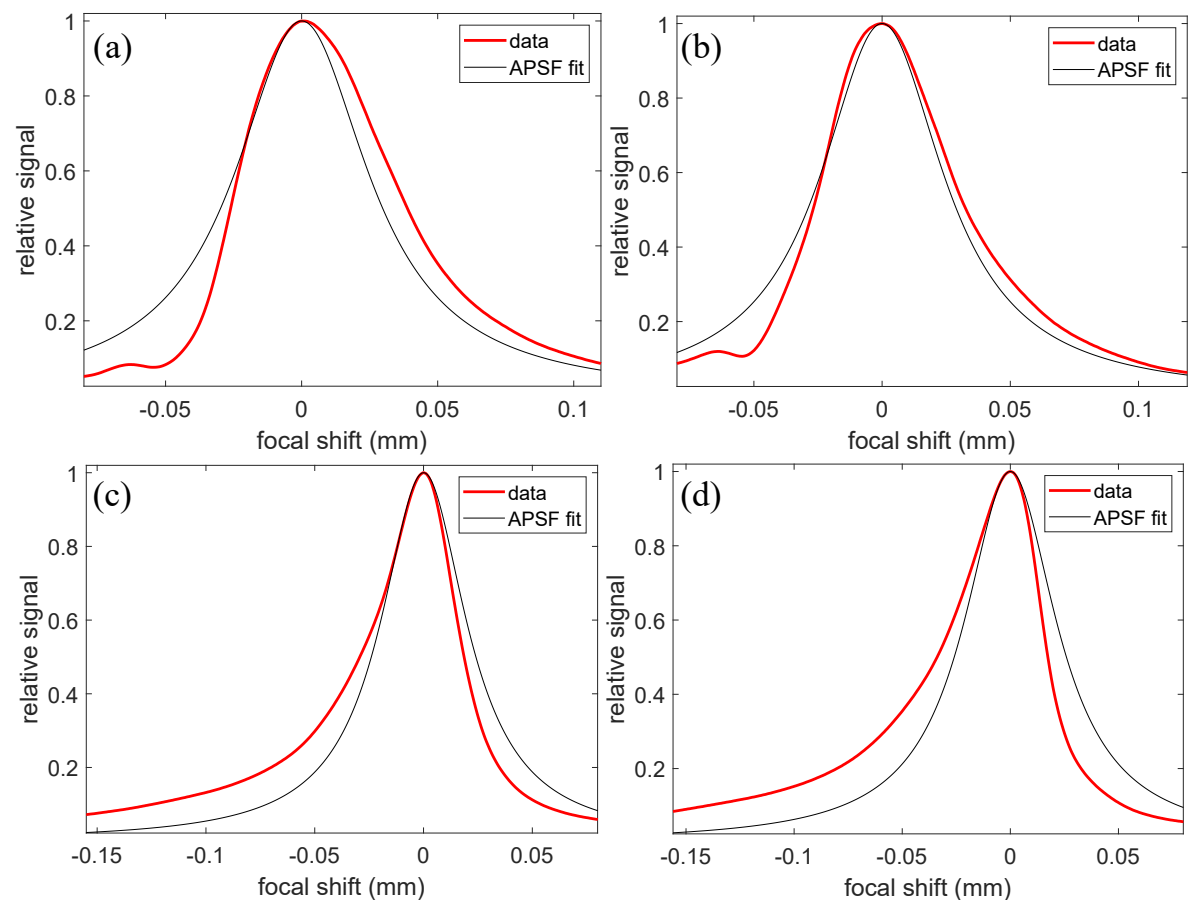

Figure 2. Experimentally obtained confocal peaks (thick red curves) with the fit to the axial point-spread function (APSF) given in equation (5) (thin black curves). The peaks shown were obtained using paired achromats (front peak (a) and rear peak (b)) and using paired aspheres (front peak (c) and rear peak (d)). The NA at the sample is 0.150 for the achromats and 0.121 for aspheres.

The OAP mirrors that were used were protected gold coated mirrors from the Thorlabs MP range. For each of the configurations tested, the collimating optic was an OAP mirror with $25.4 \mathrm{~mm}$ focal length. The focusing optics had focal lengths of $15 \mathrm{~mm}, 25.4 \mathrm{~mm}, 33 \mathrm{~mm}$, and $50.8 \mathrm{~mm}$. This resulted in optical configurations with NAs of $0.202,0.121,0.093$, and 0.061 respectively. The confocal peaks generated for each of the configurations are shown in figure 3, with the front peaks in the left column and the rear peaks in the right column. Generally, the peaks generated with each of the mirror configurations appear much more symmetric than those generated with either lens configuration and the curves are much more closely matched to the APSF fit. The exception is the rear peak generated when using the $15 \mathrm{~mm}$ focusing mirror. These peak exhibits significant distortion due to spherical aberration introduced by the front sample surface. This is due to the relatively high NA of the configuration and is a known issue in confocal microscopy where optics with even higher NA than those considered here are often used $[9,10]$.

A summary of the properties of the peaks shown in figures 2 and 3 is given in table 1 . This includes the full-width at half maximum (FWHM) of each peak and the rms (root-mean-square) error of the fit to the APSF. Reducing the system NA results in the generation of wider confocal peaks. The rms fit error provides an indication of how symmetric the peak is, with lower rms error values implying greater peak symmetry. The peaks with the largest rms fit error are those obtained with the lens configurations and the rear peak of the highest NA mirror configurations, which is consistent with the appearance of these more asymmetric peaks in figures 2 and 3. 

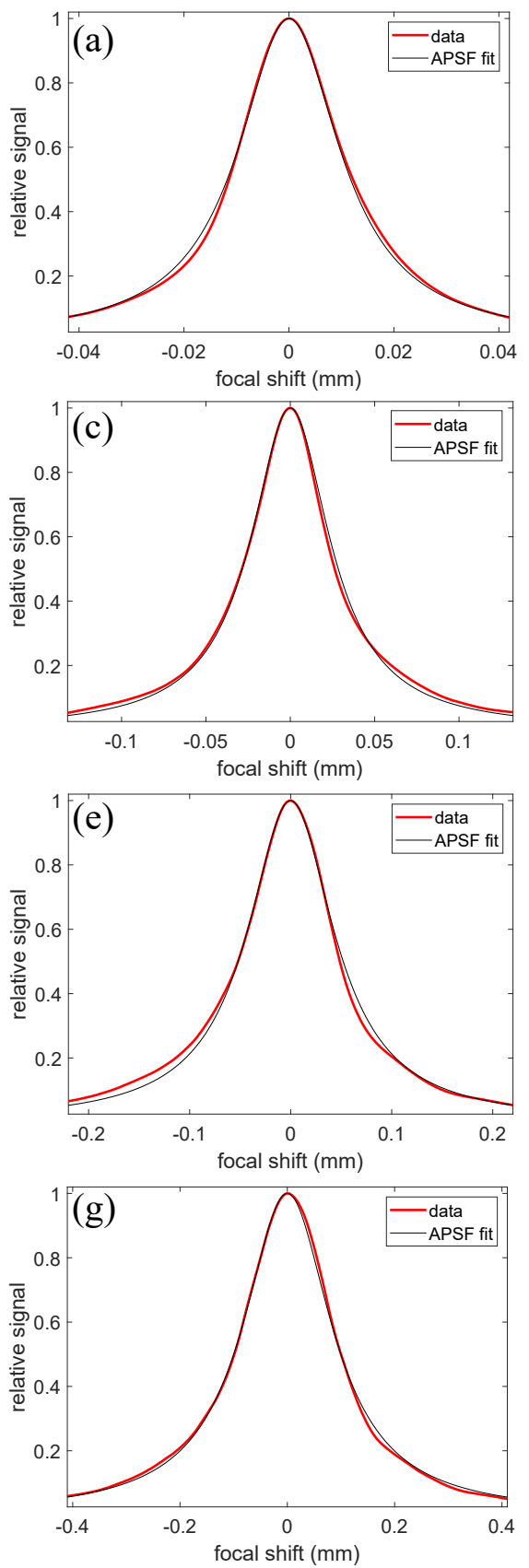
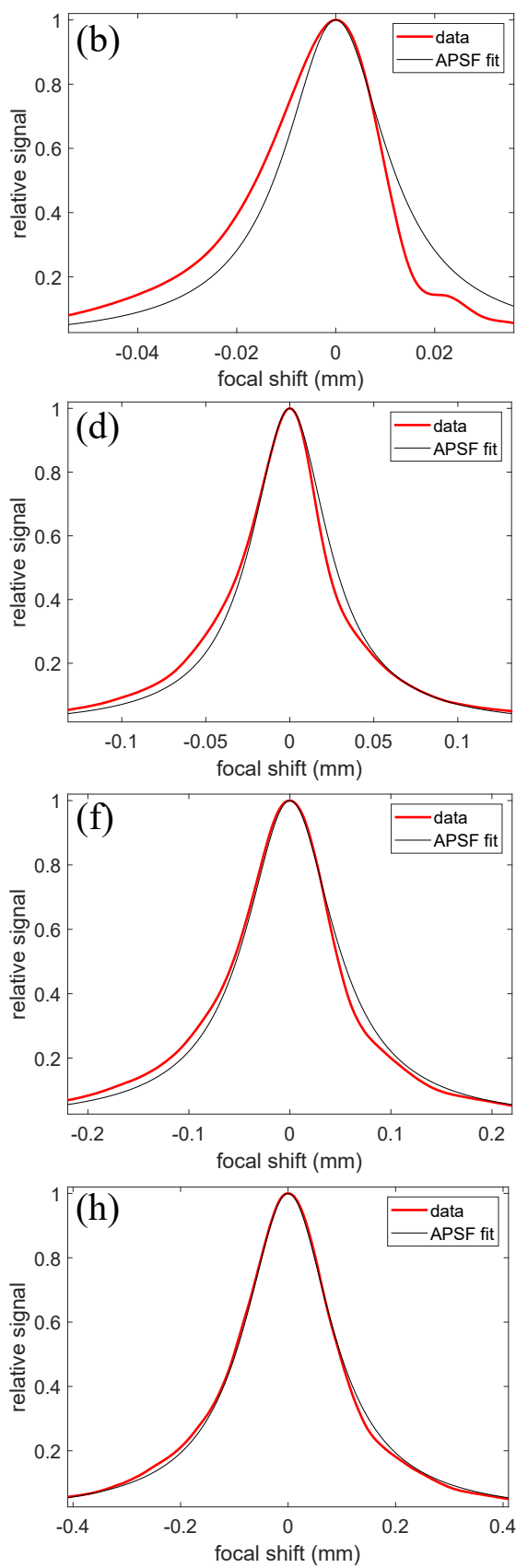

Figure 3. Experimentally obtained confocal peaks (thick red curves) with the fit to the axial PSF given in equation (5) (thin black curves) for different OAP mirror configurations. In each case the collimating optic is the same (f=25.4 mm) with a different focusing optic in each case providing an NA at the sample of 0.202 for (a) and (b), 0.121 for (c) and (d), 0.093 for $(\mathrm{e})$ and $(\mathrm{f})$, and 0.061 for $(\mathrm{g})$ and $(\mathrm{h})$. The front peaks are shown on the left and the rear peaks are shown on the right. 
Table 1. Summary of the properties of the confocal peaks obtained with different optical configurations (shown in figures 2 and 3). The error columns refer to the rms error of the fit to the axial point-spread function.

\begin{tabular}{|c|c|c|c|c|c|c|}
\hline Collimator & Focuser & $\begin{array}{l}\text { Sample } \\
\text { NA }\end{array}$ & $\begin{array}{c}\text { Front } \\
\text { FWHM }(\mu \mathrm{m})\end{array}$ & $\begin{array}{c}\text { Rear } \\
\text { FWHM }(\mu \mathrm{m})\end{array}$ & $\begin{array}{l}\text { rms error } \\
\text { (front) }\end{array}$ & $\begin{array}{c}\text { rms error } \\
\text { (rear) }\end{array}$ \\
\hline $\begin{array}{c}\text { Achromat } \\
(f=20 \mathrm{~mm})\end{array}$ & $\begin{array}{c}\text { Achromat } \\
(f=16 \mathrm{~mm})\end{array}$ & 0.150 & 64.9 & 59.8 & 0.091 & 0.051 \\
\hline $\begin{array}{c}\text { Asphere } \\
(f=11 \mathrm{~mm})\end{array}$ & $\begin{array}{c}\text { Asphere } \\
(f=11 \mathrm{~mm})\end{array}$ & 0.121 & 48 & 51.2 & 0.080 & 0.106 \\
\hline $\begin{array}{c}\text { OAP mirror } \\
(f=24.5 \mathrm{~mm})\end{array}$ & $\begin{array}{l}\text { OAP mirror } \\
(f=15 \mathrm{~mm})\end{array}$ & 0.202 & 23.7 & 26.6 & 0.013 & 0.083 \\
\hline $\begin{array}{l}\text { OAP mirror } \\
(f=24.5 \mathrm{~mm})\end{array}$ & $\begin{array}{l}\text { OAP mirror } \\
(f=24.5 \mathrm{~mm})\end{array}$ & 0.121 & 55.0 & 52.6 & 0.015 & 0.033 \\
\hline $\begin{array}{l}\text { OAP mirror } \\
(f=24.5 \mathrm{~mm})\end{array}$ & $\begin{array}{l}\text { OAP mirror } \\
(f=33 \mathrm{~mm})\end{array}$ & 0.093 & 101 & 104 & 0.019 & 0.027 \\
\hline $\begin{array}{c}\text { OAP mirror } \\
(f=24.5 \mathrm{~mm})\end{array}$ & $\begin{array}{l}\text { OAP mirror } \\
(f=50.8 \mathrm{~mm})\end{array}$ & 0.061 & 196 & 197 & 0.011 & 0.013 \\
\hline
\end{tabular}

\subsection{Refractive index and thickness measurements}

Refractive index and thickness measurements were made by scanning the sample and reference mirror to acquire the three quantities $\Delta z, \Delta l$, and $\mathrm{d} \Delta z / \mathrm{d} \nu$ introduced in section 2. To calculate the low-coherence term $\Delta l$, accurate determination of the centre of the fringe envelope is required. Extraction of the envelope from the fringe pattern was achieved using the Hilbert transform [11]. Peak centres were determined by taking the maxima of polynomial fits to the fringe envelopes. Fringe envelopes acquired from the front sample surface using the achromat pairing and the OAP mirror pairing using the $25.4 \mathrm{~mm}$ focuser are shown in figures 4(a) ands 4(b) respectively. The fringe bursts acquired with the OAP mirror pairings are broader and of lower modulation than those obtained with either lens pairing for both front and rear surfaces.

The variation of the confocal parameter obtained with the OAP mirror pairing using the $25.4 \mathrm{~mm}$ focuser is shown in figure $4(\mathrm{c})$. The gradient of this data is used to calculate the dispersion term $\mathrm{d} \Delta z / \mathrm{d} \nu$. The sample thickness and refractive index values were calculated from these quantities using equations (1) to (4). Table 2 summarises the measurements made using each of the optical configurations. The standard deviation of ten independent measurements made with each configuration is shown in the table for each of the measured quantities $n_{\mathrm{p}}, n_{\mathrm{g}}$, and $t$. The percentage error is obtained from the difference between the measured value and the reference value for each quantity. The reference refractive index values for BK7 at $840 \mathrm{~nm}$ are 1.5100 for $n_{\mathrm{p}}$ and 1.5252 for $n_{\mathrm{g}}$ [12]. The reference thickness value was obtained by measuring with a Mitutoyo MDH digital micrometer gauge with $0.1 \mu \mathrm{m}$ resolution, and was found to be $3.1230 \pm 0.0002 \mathrm{~mm}$.

Each of the OAP mirror configurations offer much better measurement consistency than either lens configuration, with the standard deviation generally being at least half that of the lens configurations. The measurement accuracy is also much better for three of the mirror configurations, although it should be noted that the measurement of $n_{\mathrm{g}}$ and $t$ is very poor for the highest NA mirror configuration. This is because of the introduction of spherical aberration by the front sample surface which causes distortion of the rear peak but not the front peak, as seen in figure 3(a). The accuracy generally improves with decreasing NA with the lowest NA configuration exhibiting the lowest percentage error. That better performance can be expected from lower NA configurations is greatly beneficial for the development of remote refractive index sensors. This is because the lower the NA at sample, the longer the standoff distance can be and thus allows larger test objects to be measured, provided the individual layer thickness is large enough to provide adequate peak separation. 

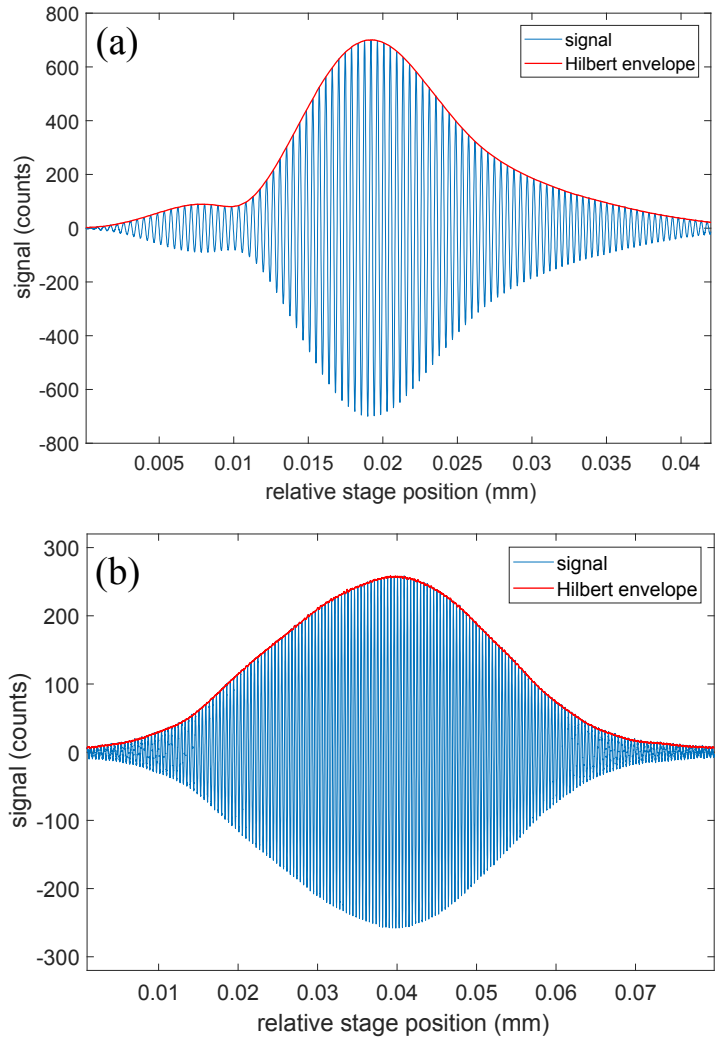

(c)

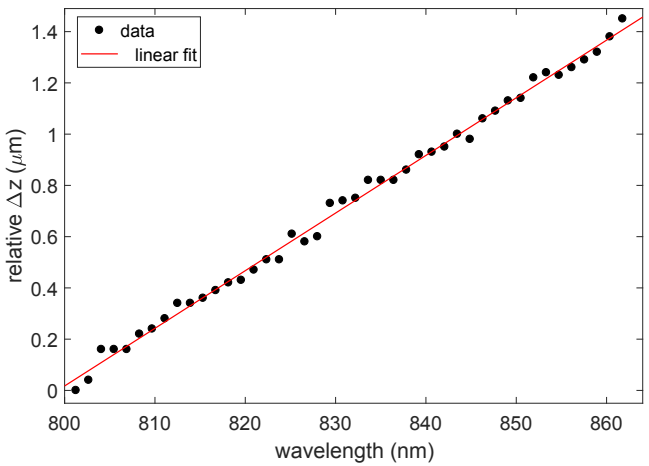

Figure 4. Low coherence fringe envelopes obtained from the front sample surface using the aspheric lens configuration (a) and the OAP mirror configuration with $25.4 \mathrm{~mm}$ focuser (b). The variation in the confocal parameter $\Delta z$ from which the dispersion data $\mathrm{d} \Delta z / \mathrm{d} \nu$ is acquired (also obtained with OAP pairing with $25.4 \mathrm{~mm}$ focuser).

Table 2. Summary of thickness and refractive index measurements for a range of different optical configurations.

\begin{tabular}{|c|c|c|c|c|c|c|c|c|c|}
\hline System & $n_{\mathrm{p}}$ & $\begin{array}{c}\text { StDev } \\
n_{\mathrm{p}}\end{array}$ & $\begin{array}{l}\text { Error } \\
(\%) n_{\mathrm{p}}\end{array}$ & $n_{\mathrm{g}}$ & $\begin{array}{c}\text { StDev } \\
n_{\mathrm{g}}\end{array}$ & $\begin{array}{l}\text { Error } \\
(\%) n_{\mathrm{g}}\end{array}$ & $t$ & $\begin{array}{c}\text { StDev } \\
t\end{array}$ & $\begin{array}{l}\text { Error } \\
(\%) t\end{array}$ \\
\hline $\begin{array}{c}\text { Paired } \\
\text { achromats }\end{array}$ & 1.5088 & 0.0012 & 0.078 & 1.5237 & 0.0013 & 0.098 & 3.1277 & 0.0028 & 0.118 \\
\hline $\begin{array}{c}\text { Paired } \\
\text { aspheres }\end{array}$ & 1.5114 & 0.0026 & 0.093 & 1.5244 & 0.0017 & 0.056 & 3.1257 & 0.0018 & 0.050 \\
\hline $\begin{array}{l}\text { OAP (15 mm } \\
\text { focuser) }\end{array}$ & 1.5094 & 0.0005 & 0.042 & 1.5188 & 0.0004 & 0.418 & 3.1382 & 0.0010 & 0.450 \\
\hline $\begin{array}{l}\text { OAP }(25.4 \mathrm{~mm} \\
\text { focuser })\end{array}$ & 1.5106 & 0.0004 & 0.039 & 1.5246 & 0.0005 & 0.038 & 3.1264 & 0.0012 & 0.072 \\
\hline $\begin{array}{l}\text { OAP (33 mm } \\
\text { focuser) }\end{array}$ & 1.5093 & 0.0005 & 0.044 & 1.5247 & 0.0004 & 0.031 & 3.1257 & 0.0010 & 0.050 \\
\hline $\begin{array}{l}\text { OAP }(50.8 \mathrm{~mm} \\
\text { focuser })\end{array}$ & 1.5103 & 0.0006 & 0.022 & 1.5255 & 0.0006 & 0.020 & 3.1238 & 0.0012 & 0.010 \\
\hline
\end{tabular}




\section{DISCUSSION AND CONCLUSIONS}

The influence of optical aberrations in the confocal channel of a combined low-coherence and confocal refractometer has been investigated. Spherical aberration distorts the axial point-spread function which causes asymmetry of the confocal peaks obtained as the object is scanned through focus. Replacing the lens system with off-axis parabolic mirrors reduces spherical aberration and results in confocal peak shapes that are much more symmetric, provided the system NA is not too great. For configurations with NAs greater than approximately 0.18 , spherical aberration introduced by the front sample surface causes significant distortion of any subsequent peaks.

Refractive index and thickness measurements are generally much better using OAP mirrors as well, with both repeatability and accuracy being at least twice as good for each of the three measured quantities for all but the highest NA configuration. Whilst improved performance can be expected for better corrected lenses, these would be more expensive than those used here. OAP mirrors therefore offer an attractive and inexpensive solution for this application. Even though the accuracy of the measurements made with high NA configuration is poor, the measurement consistency is good which suggests that accurate measurements could still be made with high NA optics if effect of the spherical aberration is corrected for. This would be beneficial for the measurement of thinner objects or multi-layered objects with a narrow separation between interfaces.

\section{REFERENCES}

[1] Sorin, W. V. and Gray, D. F., "Simultaneous thickness and group index measurement using optical lowcoherence reflectometry," IEEE Photonic. Tech. L. 4(1), 105-107 (1992).

[2] Rheims, J., Köser, J., and Wriedt, T., "Refractive index measurements in the near ir using an abbe refractometer," Meas. Sci. Technol. 8(6), 601-605 (1997).

[3] Buggy, S. J., Chehura, E., s. W. James, and Tatam, R. P., "Optical fibre refractometers for resin cure monitoring," J. Opt. A-Pure Appl. Op. 9(6), S60-S65 (2007).

[4] Fukano, M. and Yamaguchi, I., "Simultaneous measurement of thicknesses and refractive indices of multiple layers by a low-coherence confocal interference microscope," Opt. Lett. 21(23), 1942-1944 (1996).

[5] Maruyama, H., Mitsuyama, T., Ohmi, M., and Haruna, T., "Simultaneous measurement of refractive index and thickness by low coherence interferometry considering chromatic dispersion of index," Opt. Rev. 7(5), 468-472 (2000).

[6] Kim, S., Na, J., Kim, M., and Lee, B. H., "Simultaneous measurement of refractive index and thickness by combining low-coherence interferometry and confocal optics," Opt. Express 16(8), 5516-5526 (2008).

[7] Francis, D., Ford, H. D., and Tatam, R. P., "Spectrometer-based refractive index and dispersion measurement using low-coherence interferometry with confocal scanning," Opt. Express 26(3), 3604-3617 (2018).

[8] van Leeuwen, T. G., Faber, D. J., and Aalders, M. C., "Measurement of the axial point spread function in scattering media using single-mode fiber-based optical coherence tomography," IEEE J. Sel. Top. Quant. 9(2), 227-233 (2003).

[9] Sheppard, C. J. R., "Aberrations in high aperture conventional and confocal imaging systems," Appl. Opt. 27(22), 4782-4786 (1988).

[10] Hell, S., Reiner, G., Cremer, C., and Stelzer, E. H. K., "Aberrations in confocal fluorescence microscopy induced by mismatches in refractive index," J. Microsc. 169(3), 391-405 (1993).

[11] Liu, P., Groves, R. M., and Benedictus, R., "Signal processing in optical coherence tomography for aerospace material characterization," Opt. Eng. 52(3), 032201 (2013).

[12] Polyanskiy, M. N., "Refractive index database," https://refractiveindex.info (accessed 15/03/2019). 
The use of parabolic mirrors in

combined low-coherence and confocal refractive index measurement

Francis, Daniel

SPIE

Francis D, Ford HD, Hallam JM, Tatam RP. (2019) The use of parabolic mirrors in combined low-coherence and confocal refractive index measurement. Proceedings Volume 11056, Optical Measurement Systems for Industrial Inspection XI: SPIE Optical Metrology, 24-27 June 2019, Munich, Germany

https://doi.org/10.1117/12.2525986

Downloaded from Cranfield Library Services E-Repository 